\title{
No calor da idade: parâmetros de conforto térmico para idosos residentes em localidade do semiárido paraibano
}

\author{
Hot age: thermal comfort parameters for elderly \\ residents in the semi-arid region of Paraiba
}

\section{Miriam de Farias Panet \\ Virgínia Maria Dantas de Araújo \\ Eduardo Henrique Silveira de Araújo}

\section{Resumo}

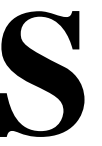

abe-se que, com o envelhecimento, acontecem alterações fisiológicas que comprometem negativamente a sensação térmica do indivíduo. No entanto, a sensação de conforto térmico de pessoas idosas em ambientes residenciais tem sido pouco explorada no Brasil, principalmente em ambientes residenciais. Nesse contexto, quais seriam os parâmetros para avaliar a qualidade térmica de ambientes residenciais para pessoas idosas? Para responder a essa questão determinaram-se os parâmetros de conforto térmico para pessoas idosas em ambiente residencial na cidade de Campina Grande, localizada no estado da Paraíba, Brasil. A pesquisa, de natureza empírica, adotou a estratégia de múltiplos métodos, com entrevista estruturada para obter os dados das variáveis individuais e subjetivas, e medição dos dados das variáveis ambientais por meio de instrumentos e equipamentos especializados. A técnica estatística de estimativa de probabilidades (probits) utilizada para determinar os parâmetros de conforto térmico comprovou a preferência das pessoas idosas por ambientes mais aquecidos. A partir dos resultados é possível contribuir com o planejamento de ambientes residenciais mais confortáveis para pessoas idosas, mediante avaliações pós-ocupação (APO) e simulações de desempenho térmico de edificações em fase de concepção projetual.

Palavras-chave: Sensação térmica. Pessoas idosas. Semiárido.

${ }^{1}$ Miriam de Farias Panet 'Universidade Federal de Campina

Grande

Campina Grande - PB - Brasil

2Virgínia Maria Dantas de Araújo ${ }^{2}$ Universidade Federal do Rio Grande do Norte Natal - RN - Brasil

${ }^{3}$ Eduardo Henrique Silveira de Araújo

${ }^{3}$ Universidade Federal do Rio Grande do Norte Natal - RN - Brasil

Recebido em 05/02/19 Aceito em 31/05/19

\section{Abstract}

Ageing, as we know, involves a series of physiological changes that have a negative impact on the individual's thermal perception. However, thermal comfort for the elderly has received little attention from researchers in Brazil, especially regardingthe elderly people's own homes. In this context, what parameters can be applied to assess thermal quality in elderly people's homes? To answer this question, thermal comfort parameters have been determined for elderly people in their homes in the city of Campina Grande, Paraiba State, Brazil. For this empirical research, a multiple-method strategy was implemented through the use of structured interviews to collect data concerning individual and subjective variables, as well as the measurement of data concerning environmental variables through the use of dedicated tools and equipment. The probit model for estimating probabilities was used to determine the parameters of thermal comfort, confirming the preference of elderly people for warmer environments. The results can help design home environments that are more comfortable for elderly people, through post-occupancy evaluation (POE) and simulations of the thermal performance of houses or building during the designing stage.

Keywords: Thermal sensation. Elderly people. Semiarid region. 


\section{Introdução}

Todas as pessoas sofrem, em certo grau, com os efeitos do calor. Porém, os grupos formados pelas crianças, pelas pessoas idosas, por pessoas acamadas com doenças crônicas, por aqueles em desvantagem socioeconômica e em ocupações específicas, como trabalhadores de fábricas ou mineradoras, se destacam como os mais vulneráveis (WORLD..., 2015).

As crianças e as pessoas idosas estão no grupo de risco principalmente por dependerem de terceiros para se adaptarem às condições ambientais e por apresentarem diferenças na produção e dissipação do calor metabólico. Para uma criança pequena, que não sabe falar, ou um idoso com demência, por exemplo, hidratar-se em um dia quente é uma tarefa que depende de um cuidador.

A ocorrência de estresse térmico, por calor, deve aumentar entre as pessoas idosas pelas seguintes razões: em primeiro lugar, devido ao aquecimento global, os eventos de ondas de calor se tornarão mais recorrentes. Em segundo lugar, a população nascida a partir da segunda metade do século XX tornou-se cada vez mais dependente do uso da climatização artificial. Em prováveis crises energéticas ou econômicas essa população sofrerá mais com a adaptação. Por último, haverá maior número de indivíduos em risco em decorrência do aumento da longevidade e do acometimento de demências (HANSEN et al., 2011).

Segundo estimativas do Instituto Brasileiro de Geografia e Estatística (INSTITUTO..., 2008), em 2050 a população relativa de pessoas com 60 anos ou mais será de 29,75\%, com expectativa de vida de 81 anos. Comparando com os dados demográficos de 2010 (19.282.049), a população de pessoas idosas irá mais que triplicar em 2050, com número de habitantes estimado em 64.050.980.

Além das razões expostas anteriormente, a sensibilidade térmica, tanto para o calor quanto para o frio, tende a declinar com o envelhecimento, principalmente nas extremidades do corpo (pernas, braços, pés, mãos, orelhas e nariz). Todavia, o declínio da sensibilidade térmica parece ser maior para as condições de calor. A pessoa idosa apresenta mais dificuldade em perceber mudanças de temperatura que tendem a aquecer o ambiente (GUERGOVA; DUFOUR, 2011).

Em experimento desenvolvido por Dufour e Candas (2007), entre grupos de pessoas com faixas etárias distintas, foi constatado maior "limite térmico relativo" das pessoas idosas em comparação às mais jovens, principalmente nas regiões das mãos, dos pés e da perna. O "limite térmico relativo" foi definido com o uso de sensores térmicos (thermode) instalados em várias regiões do corpo. Os sensores foram regulados para uma temperatura inicial neutra. No momento em que os indivíduos percebiam as variações térmicas, acionavam um botão e os resultados de temperatura eram registrados, ou seja, quanto maior fosse o limite térmico relativo, menor seria a sensibilidade do indivíduo às variações térmicas.

Conceito complementar foi desenvolvido por Mekjavic e Bligh (1989ªpud NOVIETO, 2013). Segundo os autores, o intervalo em que a ativação da vasoconstrição ou da vasodilatação está ausente é denominado de "zona térmica nula" (ZTN) de temperatura central $\left(\mathrm{T}_{\mathrm{ty}}\right)$. O comprometimento do desempenho do sistema passivo e dos órgãos do corpo humano afetados com o envelhecimento pode provocar mudança nos limites da temperatura central para início dos mecanismos de resfriamento e aquecimento do corpo, o que influencia a magnitude da "zona térmica nula" (NOVIETO, 2013).

A relação entre a ZTN de temperatura central e a zona nula, ou neutra, de sensação térmica também foi estudada por Kingma, Schweiker e Lichtenbelt (2017). Os autores consideraram o princípio de que, assim como nos mamíferos, o corpo humano se utiliza das informações passadas pela sensação térmica para a conservação de energia através de comportamentos voluntários e involuntários. Fora do alcance da ZTN a temperatura do corpo pode ser mantida, entretanto haverá gastos energéticos com o aumento do metabolismo ou perda de água através do suor. Portanto, para os mais vulneráveis os riscos à saúde para manter-se na ZTN podem ser maiores.

Nesse contexto, alguns autores, como Kenney e Munce (2003), Wilson et al. (2010) e Tsuzuki e Ohfuku (2002), afirmam que os níveis de temperatura do corpo de uma pessoa idosa saudável não são diferentes daqueles em indivíduos jovens. Quando expostos ao calor, a temperatura da pele aumenta, mas a temperatura interna do corpo permanece constante, a não ser que a exposição seja mais prolongada e que as condições de calor sejam extremas, a ponto de contribuir com um maior esforço do corpo para manter-se em equilíbrio.

1MEKJAVIC, I. B.;BLIGH, J. Core threshold temperatures for sweating. Canadian Journal of Physiology and Pharmacology, v. 67, p. 1038 1044, 1989. 
As alterações no sistema de termorregulação da pessoa idosa podem afetar também a temperatura interna basal. Gunes e Zaybak (2008) mediram a temperatura axilar de um grupo formado por 133 pessoas idosas para investigar possíveis alterações da temperatura interna e constataram que a temperatura basal média axilar foi de $35,7{ }^{\circ} \mathrm{C}$, diferente daquela adotada em análises clínicas. Assim, seria incorreto adotar a temperatura basal de $36,5^{\circ} \mathrm{C}$ como referência para pessoas idosas em diagnósticos de saúde.

$\mathrm{Na}$ investigação das variáveis idade, taxa metabólica basal, débito cardíaco e peso entre jovens e pessoas idosas, Novieto e Zhang (2010) adaptaram o modelo termofisiológico de Fiala (1998) aos parâmetros de uma pessoa idosa definida como padrão. Como esperado, o modelo que representa a pessoa idosa apresentou desconforto local, nas mãos, maior que o representado pelo modelo original. Segundo os autores, "isso pode ser atribuído aos efeitos combinados da baixa taxa metabólica, da redução da circulação sanguínea nas extremidades, e da baixa capacidade de aquecimento nas partes do corpo devido à perda de peso" (NOVIETO; ZHANG, 2010, p. 8).

Apesar dos estudos apresentados, ainda há carência de pesquisas na área de conforto térmico dedicadas a pessoas idosas. Dos modelos de conforto térmico investigados por Panet (2018) apenas o modelo termofisiológico desenvolvido por Novieto (2013) considerou as características dessa faixa etária.

$\mathrm{O}$ mesmo acontece quanto à região predominante dos estudos e quanto ao ambiente construído utilizado para tais fins. Daqueles desenvolvidos no Brasil, a maioria está concentrada em regiões de clima quente e úmido, normalmente pesquisas realizadas em ambientes escolares, de empresas ou em câmaras climatizadas, com controle das variáveis microclimáticas (ARAÚJO, 1996).

Todavia, como as pessoas idosas passam a maior parte do tempo no interior de suas residências, principalmente aquelas já aposentadas, entende-se que o estudo do conforto térmico desses indivíduos é mais indicado em ambientes residenciais. Nesses ambientes, o indivíduo está sujeito às variações climáticas e é agente ativo nas trocas térmicas com o meio circundante, através do controle pessoal dos dispositivos adaptativos, como o uso de vestimentas adequadas, o acionamento de portas e janelas, e a ativação de ventiladores ou de aparelhos de arcondicionado.

Os problemas térmicos das habitações foram apontados por Chindapol et al. (2014) como uma das lacunas existentes nas políticas públicas internacionais que visam à manutenção da saúde de pessoas idosas. Com basenessa constatação, quais seriam os parâmetros para avaliar a qualidade térmica de ambientes residenciais para pessoas idosas?

Comesse questionamento, determinaram-se parâmetros de conforto térmico para pessoas idosas em ambiente residencial na cidade de Campina Grande, PB, partindo do princípio de que esse grupo etário tende a se sentir confortável em ambientes mais aquecidos. Para tanto, investigou-se a sensação de conforto térmico desse grupo em edificações residenciais localizadas na zona urbana de Campina Grande, tendo como objeto de estudo a sensação térmica de pessoas idosas no interior de suas residências.

\section{Método}

A pesquisa, de natureza empírica, adotou a estratégia de múltiplos métodos, que, por ser constituída pela associação de dois ou mais instrumentos de investigação, garante maior confiabilidade na análise dos dados (VILLA; ORNSTEIN, 2013). Os instrumentos de investigação utilizados foram a entrevista estruturada, a medição dos dados das variáveis ambientais e o formulário de observação do ambiente. O detalhamento dos procedimentos adotados será descrito nos seguintes itens:

(a) caracterização da área de estudo;

(b) procedimentos iniciais;

(c) coleta de dados; e

(d) tratamento e análise dos dados.

\section{Caracterização da área de estudo}

O município de Campina Grande está inserido na mesorregião do Agreste Paraibano, com latitude de $07^{\circ} 13$ '50" e longitude de $35^{\circ} 52^{\prime} 52^{\prime}$ '. Está a aproximadamente $120 \mathrm{~km}$ da cidade de João Pessoa, capital da Paraíba, e se encontra entre microrregiões de clima e vegetação diferentes. Apesar de apresentar clima tropical com estação seca (As), o município caracteriza-se por temperatura média anual de $23,5{ }^{\circ} \mathrm{C}$ (INSTITUTO..., 2016), devido a sua altitude de aproximadamente $555 \mathrm{~m}$ acima do nível do mar 
(OLIVEIRA, 2013). Na nova proposta de zoneamento bioclimático para o Brasil, Campina Grande se encontra na zona bioclimática de número 10 , no grupo de temperaturas médias anuais entre $20^{\circ} \mathrm{C}$ e $26^{\circ} \mathrm{C}$ (RORIZ, 2012). Os dados das estatísticas das normais climatológicas para a cidade de Campina Grande (INSTITUTO..., 2018b) estão apresentados na Tabela 1.

O ano de 2016, período de coleta de dados, caracterizou-se como atípico por apresentar precipitação pluviométrica acumulada de 435,5 mm (INSTITUTO..., 2018a).

\section{Procedimentos iniciais}

Os participantes da pesquisa foram pessoas com idade superior a 60 anos, residentes na cidade de Campina Grande, não acamadas e com saúde física e mental, ou seja, sem mobilidade comprometida e capazes de executar com autonomia todas as atividades da vida diária.

As residências das pessoas participantes da pesquisa foram em sua totalidade do tipo unifamiliar, térreas, com paredes em alvenaria de tijolos cerâmicos e cobertas com telhas cerâmicas do tipo canal. Os sistemas construtivos não foram levados em conta nas análises realizadas porque a sensação térmica dos pesquisados já se trata do resultado da interação das condições ambientais locais com a envoltória da construção; portanto, embora não sejam o objetivo da pesquisa, poderão ser analisados em etapa posterior.

As residências estão localizadas em bairros populares e foram selecionadas de forma a garantir uma distribuição espacial homogênea, dentro dos limites da zona urbana.

O período de coleta dos dados ocorreu entre os meses de abril e dezembro de 2016, de modo a abranger todas as variações climáticas anuais da região objeto de estudo.

O tamanho preliminar da amostra foi estimado com as técnicas estatísticas de amostragem por estratos e amostragem aleatória simples. Os distritos sanitários foram adotados como estratos.

Os serviços de atendimento à saúde básica estão setorizados, na zona urbana de Campina Grande, em seisdistritos sanitários (DS), com suas unidades básicas de saúde (UBS), que atendem a um número variado de habitantes dos bairros circunvizinhos, a depender do número de equipes de saúde da família (ESF) disponíveis nas UBS (BRASIL, 2006). A Tabela 2 apresenta o número de bairros que integram cada DS em Campina Grande.

De cada DS foram visitadas quatro UBS, localizadas em bairros distintos. De cada UBS, a partir da amostragem aleatória simples, selecionou-se uma média de 10 participantes. A frequência absoluta e relativa do total de participantes por distrito sanitário está apresentada na Tabela 3.

No estudo piloto foram entrevistadas 32 pessoas idosas moradoras do condomínio Cidade Madura, localizado no bairro Cidade Verde II. Após análise preliminar dos resultados, percebeu-se que o medidor de estresse térmico utilizado apresentava problema. Assim, depois de investigações acerca do estado dos equipamentos disponíveis, decidiu-se pela aquisição de um novo medidor certificado e calibrado. Ao todo foram entrevistadas 340 pessoas idosas, das quais 32 foram descartadas, totalizando 308 participantes.

Na sequência, a Tabela 4 mostra a lista dos bairros visitados durante a pesquisa de campo em cada DS na cidade de Campina Grande.

\section{Coleta de dados}

Os procedimentos iniciais de seleção dos participantes, o acesso a suas residências e a quantificação das variáveis contaram com a colaboração dos agentes comunitários de saúde (ACS), locados nas UBS selecionadas. Para tanto,foi necessária a obtenção de autorização formal na Secretaria de Saúde do Município, com o gerente de atenção básica. No ato da visita à Secretaria foram fornecidos pelo gerente a autorização formal e os contatos de todas as UBS.

Os procedimentos realizados foram aprovados pelo Comitê de Ética em Pesquisa com Seres Humanos (CEP/UFCG), sob o projeto de número CAAE 52584315.7.0000.5182 e parecer de número 1.872.415.

Nas primeiras visitas às UBS contatou-se a médica/enfermeira-chefe de cada UBS para apresentação da pesquisa e indicação de um ACS. Em seguida, apresentavam-se as intenções da pesquisa ao ACS para definição das residências a serem visitadas e o período de duração das visitas. A seleção das pessoas idosas contou com o conhecimento da comunidade por parte dos ACS. 
Tabela 1 - Estatísticas das normais climatológicas da cidade de Campina Grande no período de 1981 2010

\begin{tabular}{r|l}
\hline Variáveis & Média Anual \\
\hline \multirow{3}{*}{ Temperatura do ar } & Máxima: $28,8^{\circ} \mathrm{C}$ \\
\cline { 2 - 2 } & Média: $23,5^{\circ} \mathrm{C}$ \\
\cline { 2 - 2 } & Mínima: $20,2^{\circ} \mathrm{C}$ \\
\hline Umidade relativa & $77 \%$ \\
\hline Velocidade do vento & $3,8 \mathrm{~m} / \mathrm{s}$ \\
\hline Direção do vento & $\mathrm{SE}-116,9^{\circ}$ \\
\hline Precipitação pluviométrica & $777 \mathrm{~mm}$ \\
\hline
\end{tabular}

Tabela 2 - Número de bairros por distrito sanitário

\begin{tabular}{c|c|c|c|c|c|c}
\hline & DS I & DS II & DS III & DS IV & DS V & DS VI \\
\hline Número de bairros/DS & 7 & 4 & 9 & 7 & 9 & 4 \\
\hline
\end{tabular}

Tabela 3 - Frequência absoluta e relativa dos participantes por distrito sanitário

\begin{tabular}{c|c|c|c|c|c|c}
\hline $\begin{array}{c}\text { Distrito } \\
\text { Sanitário } \\
\text { I }\end{array}$ & $\begin{array}{c}\text { Distrito } \\
\text { Sanitário } \\
\text { II }\end{array}$ & $\begin{array}{c}\text { Distrito } \\
\text { Sanitário } \\
\text { III }\end{array}$ & $\begin{array}{c}\text { Distrito } \\
\text { Sanitário } \\
\text { IV }\end{array}$ & $\begin{array}{c}\text { Distrito } \\
\text { Sanitário } \\
\text { V }\end{array}$ & $\begin{array}{c}\text { Distrito } \\
\text { Sanitário } \\
\text { VI }\end{array}$ & Total \\
\hline $\mathbf{( 6 2 ) \mathbf { 2 0 \% }}$ & $(55) 18 \%$ & $(40) 13 \%$ & $(65) 21 \%$ & $(43) 14 \%$ & $(43) 14 \%$ & $(308) 100 \%$ \\
\hline
\end{tabular}

Tabela 4 - Relação dos bairros visitados em cada distrito sanitário

\begin{tabular}{c|c|c|c|c|c|c}
\hline & $\begin{array}{c}\text { Distrito } \\
\text { Sanitário I }\end{array}$ & $\begin{array}{c}\text { Distrito } \\
\text { Sanitário II }\end{array}$ & $\begin{array}{c}\text { Distrito } \\
\text { Sanitário III }\end{array}$ & $\begin{array}{c}\text { Distrito } \\
\text { Sanitário IV }\end{array}$ & $\begin{array}{c}\text { Distrito } \\
\text { Sanitário V }\end{array}$ & $\begin{array}{c}\text { Distrito } \\
\text { Sanitário VI }\end{array}$ \\
\hline \multirow{2}{*}{$\underset{*}{*} \begin{array}{c}\text { Jardim } \\
\text { Tavares }\end{array}$} & Bodocongó & Alto Branco & Estação Velha & Três Irmãs & Ramadinha \\
\cline { 2 - 7 } & Monte Castelo & Bela Vista & Jeremias & Tambor & Santa Cruz & Malvinas \\
\cline { 2 - 7 } & José Pinheiro & Mutirão & Monte Santo & Santa Terezinha & Ligeiro & Serrotão \\
\cline { 2 - 7 } & Nova Brasília & Pedregal & Conceição & Catolé & Velame & Jardim Verdejante \\
\hline
\end{tabular}

A coleta de dados durou 39 dias e foram entrevistadas em média 8 pessoas por dia, sendo apenas 1 participante por residência. Essa medida procurou evitar a possível influência das respostas por parte de outro morador.

A Tabela 5 mostra o total de dias de duração da coleta de dados distribuídos por mês e o número de participantes.

Os procedimentos de coleta de dados ocorreram da forma a seguir.

Durante a visita domiciliar o ACS apresentava a pesquisadora à pessoa idosa (participante) e explicava de forma sucinta o objetivo da visita. Em seguida, a pesquisadora explicava os procedimentos de forma mais detalhada e oferecia o termo de consentimento livre e esclarecido (TCLE) para que o participante pudesse ler com calma e, se estivesse de acordo, assinar. De posse do TCLE assinado, a coleta de dados era iniciada.

A coleta de dados ocorreu na sala de estar da residência de cada pessoa idosa selecionada, no período da manhã. Durante os procedimentos os participantes se mantiveram sentados e em repouso.

Caso o participante não estivesse em repouso a mais de $30 \mathrm{~min}$ antes do início da coleta de dados, assumiam-se duas posições: (1) aguardava-se o prazo necessário para sua aclimatação; ou (2) partia-se para o próximo da lista e, depois, retornava-se. Ao final da manhã, caso o mínimo de participantes definido por UBS não fosse atingido, retornava-se ao local em outra data para a conclusão da lista original, ou uma lista complementar era selecionada para posterior aplicação.

No início das apresentações e conversas informais a pesquisadora instalava os equipamentos nos locais mais adequados e próximos ao participante, a 0,60m de altura do piso, equivalente ao nível do abdômen de uma pessoa sentada, considerando o ambiente residencial do tipo homogêneo, Classe C (INTERNATIONAL..., 1998). 
Com o medidor de estresse térmico, modelo ITWTG 2000, marca Instrutemp, foram coletados os dados da temperatura de bulbo seco (TBS), da temperatura de globo $\left(\mathrm{T}_{\mathrm{g}}\right)$ e da umidade relativa do ar (UR). Esse equipamento tem faixas de temperatura de $0{ }^{\circ} \mathrm{C}$ a $50{ }^{\circ} \mathrm{C}$ com precisão de $\pm 0,6{ }^{\circ} \mathrm{C}$ e umidade relativa de $0 \%$ a $100 \%$ com precisão de $\pm 3 \%$. Para a medição dos dados da velocidade do ar $\left(\mathrm{V}_{\mathrm{ar}}\right)$ foi utilizado o termoanemômetro digital modelo TAR-176, da marca Instrutherm, com resolução de $0,01 \mathrm{~m} / \mathrm{s}$ e precisão de $\pm(2 \%+0,2 \mathrm{~m} / \mathrm{s})$.

Os dados das variáveis ambientais foram registrados a cada $5 \mathrm{~min}$, com duração média total dos procedimentos de 30 min para cada participante (Figura 1).

O questionário utilizado para a coleta dos dados foi testado no estudo piloto, e, de acordo com o resultado, algumas questões foram alteradas para facilitar a compreensão do participante.

As primeiras questões apresentadas aos participantes foram com relação às variáveis individuais sexo, idade, peso e altura. $\mathrm{O}$ sexo e a idade foram registrados pelas categorias apresentadas na Tabela 6.

A altura e o peso eram perguntados aos participantes. Quando o participante não sabia responder, a informação era obtida na UBS.

Para a determinação dos valores da vestimenta foi adotada a forma indireta, que consiste no somatório dos valores de cada peça de roupa do vestuário (INTERNATIONAL..., 2007). Assim, durante a coleta de dados, a composição da vestimenta era anotada pela pesquisadora na planilha de peças individuais do vestuário, com seus respectivos valores em clo, de acordo com a norma ISO 7730 (INTERNATIONAL..., 2005). Como os participantes foram abordados no interior de suas casas, algumas peças do vestuário como roupas de trabalhos, por exemplo, foram desprezadas na elaboração dos itens da planilha.

No formulário de observação do ambiente era registrado o comportamento dos participantes quanto ao uso das oportunidades adaptativas presentes no cômodo utilizado (janelas, portas, ventiladores e aparelhos de arcondicionado). Nesse caso, era observado se essas oportunidades estavam presentes no ambiente, se estavam sendo utilizadas (no caso de portas e janelas, se estavam abertas) e se eram de fácil acesso do participante.

As questões subjetivas foram introduzidas na sequência, de acordo com a escala de 7 pontos. Foi perguntado ao participante como ele estava se sentindo naquele momento, e as opções de respostas eram as seguintes: (1) com muito calor, (2) com calor, (3) com pouco calor, (4) nem calor nem frio, (5) com pouco frio, (6) com frio e (7) com muito frio. A sequência dos procedimentos de coleta de dados teve como objetivo alcançar o maior tempo possível para uma melhor aclimatação do participante.

Além dos dados coletados em campo, a temperatura radiante média (TRM), o índice de massa corporal (IMC) e a taxa metabólica foram calculados.

Para o cálculo da TRM são necessários os dados da temperatura e velocidade do ar, da temperatura de globo e da emissividade do globo ( $\mathrm{gg}$ ). Teoricamente, o globo pode ter qualquer diâmetro, entretanto com globos de diâmetros diferentes de $15 \mathrm{~cm}$ devem-se adotar a equação cujo diâmetro do globo conste em suas variáveis a fim de evitar distorções nos dados. Na presente pesquisa o medidor de estresse térmico utilizado possuía globo com 7,5 cm de diâmetro e emissividade igual a 0,95.

A equação apresentada a seguir (Equação 1), recomendada pela norma ISO 7726 (1998), parte do princípio do balanço das trocas térmicas entre o globo e o ambiente ao seu redor, considerando a convecção forçada.

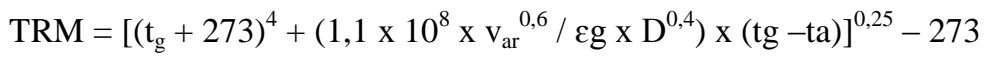

Eq. 1

O IMC foi calculado pela razão entre o peso e o quadrado da altura, e analisado segundo as categorias "baixo peso", "eutrofia" e "sobrepeso" definidas para pessoas idosas (Tabela 7).

Tabela 5 - Síntese do total de dias de coleta de dados por mês e número de participantes por mês

\begin{tabular}{c|c|c|c|c|c|c|c|c|c|c}
\hline $\begin{array}{c}\text { Número de } \\
\text { dias/mês }\end{array}$ & abr & mai & jun & jul & ago & set & out & nov & dez & total \\
\cline { 2 - 10 } & 07 & 04 & 0 & 02 & 07 & 03 & 07 & 05 & 04 & 39 \\
\hline $\begin{array}{c}\text { Número de } \\
\text { participantes/mês }\end{array}$ & 51 & 26 & 0 & 18 & 63 & 22 & 62 & 42 & 24 & 308 \\
\hline
\end{tabular}


Figura 1 - Linha do tempo da coleta de dados

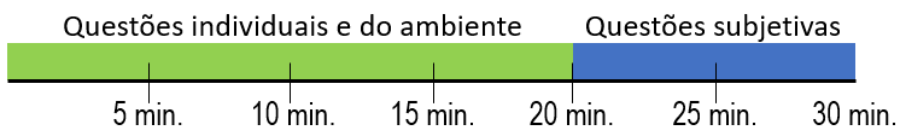

Tabela 6 - Categorias das variáveis sexo e idade

\begin{tabular}{|c|c|}
\hline Sexo & Idade \\
\hline (1) Feminino & $\begin{array}{l}\text { (1) } 60 \text { a } 70 \text { anos } \\
\text { (2) } 71 \text { a } 80 \text { anos }\end{array}$ \\
\hline (2) Masculino & $\begin{array}{l}\text { (3) } 81 \text { a } 90 \text { anos } \\
\text { (4) mais de } 90 \text { anos }\end{array}$ \\
\hline
\end{tabular}

Tabela 7 - Valores de referência do IMC

\begin{tabular}{c|c}
\hline Situação & IMC - valores de referência \\
\hline Baixo peso & Abaixo de 22 \\
Eutrofia & 22 a 27 \\
Sobrepeso & Acima de 27 \\
\hline
\end{tabular}

Fonte: adaptada de Lipschitz (1994).

Os valores da taxa metabólica foram determinados, como sugerido pela norma ISO 8996 (INTERNATIONAL..., 2004), pela categoria de observação, nível 2, método 2B. O método consiste no produto dos seguintes valores estimados: taxa metabólica basal (TMB) + taxa metabólica pelo tipo de postura + taxa metabólica pelo tipo de trabalho + taxa metabólica pelo movimento do corpo durante a atividade. Todos esses valores, com exceção da TMB, estão tabelados na referida norma. Os dados da TMB foram obtidos por Mountcastle (1980² apud FERREIRA, 2001), como apresentado na Tabela 8.

\section{Tratamento e análise dos dados}

Os procedimentos de análise dos dados foram compostos pelas análises estatísticas gerais e pelas análises estatísticas inferenciais. As análises estatísticas gerais consistiram nas análises exploratória e descritiva de dados das variáveis medidas em campo e calculadas (IMC, TRM e taxa metabólica). A análise exploratória foi realizada com o objetivo de revisar os dados registrados nos formulários e descobrir possíveis outliers, eliminando-os. Os outliers são entendidos como dados discrepantes, que por algum motivo não correspondem ao padrão dos dados.

A análise descritiva consistiu no cálculo das frequências absolutas e relativas das variáveis individuais e subjetivas, e no cálculo das estatísticas (média, mediana, máxima, mínima, desvio padrão e coeficiente de variação) das variáveis ambientais.

Nas análises estatísticas inferenciais a associação entre a variável "grau de sensação térmica" e as variáveis ambientais foi analisada mediante dois procedimentos:

(a) análise gráfica do cruzamento entre as variáveis; e

(b) análise multivariada, para testar a comparação múltipla de médias, utilizando o teste de Tukey-HSD, em que os resultados foram obtidos na ANOVA.

Já as associações entre as variáveis individuais categóricas nominais e a variável "grau de sensação térmica" foram analisadas através da técnica de análise de dados categorizados, em que foi utilizado o teste de hipótese do tipo Qui-Quadrado, com um nível de significância de $\alpha=5 \%$.

As categorias do grau de sensação térmica "muito calor", “calor" e "pouco calor", de um lado, e "pouco frio, frio", de outro, foram concatenadas, dando origem às categorias "quente" e "frio". A categoria de sensação térmica "nem calor, nem frio" deu origem à categoria "confortável".

${ }^{2}$ MOUNTCASTLE, V.B. Medical physiology. 14. ed. St. Louis: C.V. Mosby Company, 1980. 
Tabela 8 - Valores da taxa metabólica basal considerando a idade e o sexo

\begin{tabular}{c|c|c}
\hline Idade (anos) & Homens $\left(\mathbf{w} / \mathbf{m}^{2}\right)$ & Mulheres $\left(\mathbf{w} / \mathbf{m}^{2}\right)$ \\
\hline $\mathbf{1 4}$ a 16 & 53,5 & 50,0 \\
$\mathbf{1 6}$ a 18 & 50,0 & 46,5 \\
$\mathbf{1 8}$ a 20 & 47,7 & 44,2 \\
$\mathbf{2 0}$ a 30 & 45,9 & 43,0 \\
$\mathbf{3 0}$ a 40 & 45,9 & 42,4 \\
$\mathbf{4 0}$ a 50 & 44,8 & 41,9 \\
$\mathbf{5 0}$ a 60 & 43,6 & 40,7 \\
$\mathbf{6 0}$ a 70 & 42,4 & 39,5 \\
$\mathbf{7 0}$ a 80 & 41,3 & 38,4 \\
\hline
\end{tabular}

Fonte: adaptada de Ferreira (2001).

Nota: ${ }^{1}$ os valores da taxa metabólica basal para a categoria de faixa etária entre 70 a 80 foram adotados para as faixas etárias superiores a 80 anos.

Os dados das novas categorias do "grau de sensação térmica" (quente, confortável e frio) foram transformados em probits e analisados com os resultados das variáveis ambientais através dos gráficos das curvas de probits acumulados.

As estimativas de probabilidades (probits) são utilizadas para calcular a porcentagem de dada população exposta a certo tipo de consequência de um efeito adverso. Em termos matemáticos, probit são as probabilidades calculadas por uma função linear.

Essas estimativas têm como base a frequência de resposta binária (sim ou não). No caso em questão, a resposta binária seria com relação à presença ou ausência de conforto. Por exemplo, as respostas podem ser para a sensação "confortável" ("sim" para conforto) ou "desconfortável" devido ao frio ou ao calor ("não" para conforto). Então, pode-se afirmar que haverá conforto quando a resposta for "confortável" e que não haverá conforto quando a resposta for "frio" ou "quente".

$\mathrm{Na}$ presente análise, como as demais categorias de sensação térmica foram concatenadas, dando origem a apenas três categorias (quente, confortável e frio), as categorias "pouco calor" e "pouco frio" foram consideradas como desconfortáveis, contrariando o que muitos autores adotam como conforto térmico a variação entre -1 e +1 (levemente frio e levemente quente).

\section{Resultados e discussões}

$\mathrm{Na}$ análise exploratória e descritiva, após a eliminação dos outliers, o banco de dados foi constituído pelas respostas de um total de 270 participantes, sendo $78 \%$ do sexo feminino e $22 \%$ do masculino. De forma geral, o número expressivo de mulheres pode refletir dois fatos: o primeiro é que as mulheres vivem mais que os homens; e o segundo representa uma população onde as mulheres se ocupam mais dos afazeres domésticos que os homens, estando presentes em casa durante o período de coleta dos dados.

Os valores da altura dos participantes variaram entre $1,35 \mathrm{~m}$ e $1,79 \mathrm{~m}$, e seu peso entre $35 \mathrm{~kg}$ e $135 \mathrm{~kg}$.

A distribuição absoluta e relativa da idade dos participantes está representada na Tabela 9.

Com os resultados apresentados na Tabela 9, pode-se concluir que a categoria entre 60 e 70 anos apresentou maior frequência entre as mulheres; entre os homens foi a categoria de 71 a 80 anos. Ratificando o que foi mencionado acima sobre os diferentes hábitos entre os diferentes sexos, pode-se supor que os homens mais jovens (entre 60 e 70 anos) não se encontravam em casa por estarem ainda em idade ativa, capazes de trabalhar para complementar a renda familiar. No caso dos mais velhos (entre 71 e 80 anos), a maioria já estava aposentada.

$\mathrm{Na}$ Tabela 10 apresentam-se as frequências absolutas e relativas dos valores calculados do IMC e suas respectivas situações. As frequências são apresentadas pelos valores totais dos participantes e por sexo.

Os dados da Tabela 10 mostram resultados preocupantes, principalmente entre as mulheres. No total, 60\% das mulheres estão em situação de sobrepeso. Esse fato reflete os maus hábitos alimentares de populações de baixa renda, como também as alterações fisiológicas próprias da idade. Em geral, a pessoa idosa perde massa magra (músculos) e ganha massa gorda, com especial destaque para as mulheres. 
Existe também uma relação entre os resultados do IMC e as respostas para o "grau de sensação térmica" apresentados na Figura 2.

Analisando os resultados do cruzamento entre as frequências relativas do "grau de sensação térmica" e do IMC dos participantes (Figura 2), percebe-se que há tendência a um maior número de pessoas com sobrepeso na categoria "quente" e, de forma contrária, com aquelas em situação de baixo peso para a resposta à categoria "frio". Esses resultados não foram analisados em separado para homens e mulheres porque a variável sexo não apresentou efeito significante sobre a sensação térmica $(\mathrm{p}>0,05)$, no teste de hipótese do Qui-Quadrado. Por outro lado, observou-se que as variáveis idade e IMC influenciaram as respostas da variável "grau de sensação térmica" (ST), pois apresentaram valores de p<0,05. Esses resultados estruturaram um modelo multinominal ordinal para ajustes das curvas entre ST e cada variável ambiental (TBS, TRM, UR e $\mathrm{V}_{\mathrm{ar}}$ ), correlacionadas com as variáveis categóricas nominais (idade e IMC).

A Tabela 11 mostra os valores das estatísticas descritivas do isolamento térmico da vestimenta, em clo. A vestimenta mais comum entre as mulheres consistiu na composição entre camiseta, bermuda ou saia, e sandália. Entre os homens, a camiseta com calça leve ou bermuda foram as mais comuns.

Sobre as oportunidades adaptativas foi observado que, dos ambientes visitados, 179 (66\%) possuíam janelas e portas para o exterior abertas durante a coleta de dados, o que favorece a ventilação cruzada, 7 (3\%) possuíam ventiladores, mas apenas $1(0,4 \%)$ estava sendo operado, e nenhum aparelho de ar condicionado foi encontrado. Todas as aberturas encontravam-se acessíveis ao participante para manuseio. Contudo, observou-se que alguns moradores preferiam deixar as janelas fechadas por questões de privacidade e segurança.

Os resultados da variável subjetiva "grau de sensação térmica" são apresentados a seguir, na Tabela 12, que mostra a distribuição da frequência absoluta e relativa.

Observa-se que a maioria dos participantes se encontrava no grau de sensação térmica relativo a "confortável". Observando os resultados das frequências relativas para "quente" e "frio" entre ambos os sexos, percebe-se que as mulheres sentem mais calor e os homens mais frio.

As frequências relativas dos dados da variável "grau de sensação térmica" mostradas na Tabela 12 foram cruzadas com as faixas etárias e apresentadas no gráfico de sobreposição de barras, na Figura 3.

A Figura 3 mostra que há tendência à redução da sensação "confortável” e aumento da sensação "frio" com o avanço da idade. Pelos resultados apresentados no gráfico, aqueles com idade superior a 90 anos apresentam tendência a perceber mais a sensação de frio que os demais participantes.

Embora o gráfico de sobreposição de barras mostre essa tendência à redução da sensação de calor pelo grupo com idade superior a 90 anos, observando os valores das estatísticas da TBS por faixa etária (Tabela 13), percebe-se que a média de TBS ocorrida para as pessoas com idade superior a 90 anos foi inferior àquelas ocorridas nos demais grupos. Assim, nesse caso, pode-se dizer que a tendência se confirma apenas entre as três primeiras faixas etárias, por serem passíveis de comparação quanto à variável TBS.

Tabela 9 - Distribuição da frequência absoluta e relativa da faixa etária dos participantes

\begin{tabular}{c|c|c|c|c|c|c}
\hline \multirow{2}{*}{$\begin{array}{c}\text { Categoria } \\
\text { (anos) }\end{array}$} & \multicolumn{2}{|c|}{ Total } & \multicolumn{2}{c|}{ Feminino } & \multicolumn{2}{c}{ Masculino } \\
\cline { 2 - 7 } Absoluta & Relativa (\%) & Absoluta & Relativa (\%) & Absoluta & Relativa (\%) \\
\hline $\mathbf{6 0}$ a 70 & 126 & 47 & 104 & 49,3 & 22 & 37,2 \\
$\mathbf{7 1 ~ a ~ 8 0}$ & 107 & 40 & 79 & 37,4 & 28 & 47,4 \\
81 a 90 & 30 & 11 & 23 & 11 & 7 & 12 \\
Maior de 90 & 7 & 2 & 5 & 2,3 & 2 & 3,4 \\
\hline Total & 270 & 100 & 211 & 100 & 59 & 100 \\
\hline
\end{tabular}

Tabela 10 - Distribuição da frequência absoluta e relativa do IMC dos participantes

\begin{tabular}{c|c|c|c|c|c|c}
\hline \multirow{2}{*}{ Situação } & \multicolumn{2}{|c|}{ Total } & \multicolumn{2}{c|}{ Feminino } & \multicolumn{2}{c}{ Masculino } \\
\cline { 2 - 7 } & Absoluta & Relativa (\%) & Absoluta & Relativa (\%) & Absoluta & Relativa (\%) \\
\hline Baixo peso & 30 & 11 & 19 & 9 & 11 & 18,6 \\
Eutrofia & 87 & 32 & 66 & 31 & 21 & 35,6 \\
Sobrepeso & 153 & 57 & 126 & 60 & 27 & 45,8 \\
\hline Total & 270 & 100 & 211 & 100 & 59 & 100 \\
\hline
\end{tabular}


Figura 2 - Gráfico do cruzamento da frequência relativa do grau de sensação térmica com os resultados do IMC

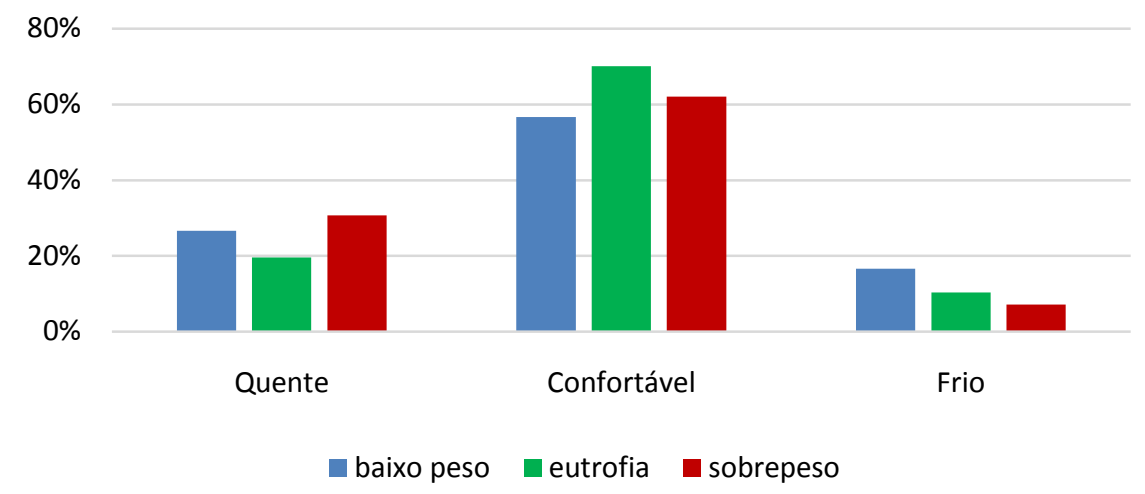

Tabela 11 - Estatísticas do isolamento térmico da vestimenta (clo) dos participantes

\begin{tabular}{c|c|c}
\hline & Feminino & Masculino \\
\hline Média & 0,32 & 0,34 \\
Mediana & 0,26 & 0,28 \\
Máxima & 0,76 & 0,75 \\
Mínima & 0,20 & 0,23 \\
Desvio padrão & 0,09 & 0,10 \\
Coeficiente de variância (\%) & 0,01 & 0,01 \\
\hline
\end{tabular}

Tabela 12 - Distribuição da frequência absoluta e relativa do grau de sensação térmica dos participantes

\begin{tabular}{c|c|c|c|c|c|c}
\hline \multirow{2}{*}{ Grau de } & \multicolumn{2}{|c|}{ Total } & \multicolumn{2}{c|}{ Feminino } & \multicolumn{2}{c}{ Masculino } \\
\cline { 2 - 7 } sensação & Absoluta & Relativa (\%) & Absoluta & Relativa (\%) & Absoluta & Relativa (\%) \\
\hline Quente & 72 & 26,7 & 59 & 27,9 & 13 & 22 \\
Confortável & 173 & 64,1 & 134 & 63,5 & 39 & 66 \\
Frio & 25 & 9,2 & 18 & 8,6 & 7 & 12 \\
Total & 270 & 100 & 211 & 100 & 59 & 100 \\
\hline
\end{tabular}

Figura 3 - Distribuição da frequência relativa do grau de sensação térmica para cada faixa etária

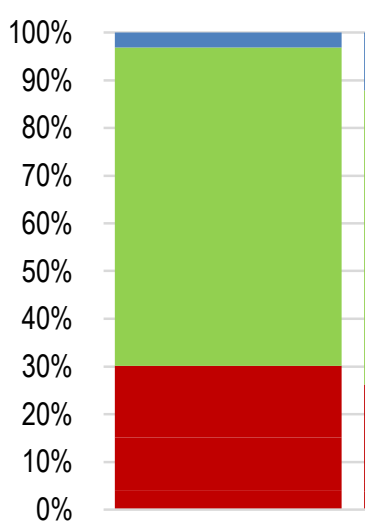

60 a 70 anos

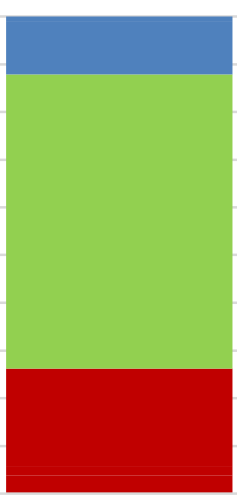

71 a 80 anos

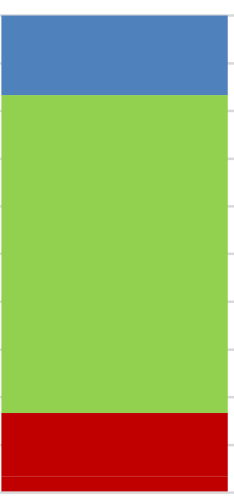

81 a 90 anos

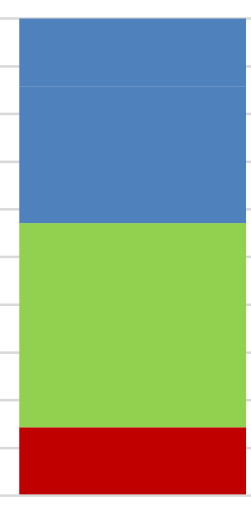

maior de 90 anos

- Quente Confortável $\quad$ Frio 
Tabela 13 -Estatísticas da TBS por faixa etária

\begin{tabular}{c|c|c|c}
\hline Faixa etária (anos) & $\begin{array}{c}\text { Média } \\
\text { TBS }\left({ }^{\circ} \mathbf{C}\right)\end{array}$ & $\begin{array}{c}\text { Mediana } \\
\text { TBS }\left({ }^{\circ} \mathbf{C}\right)\end{array}$ & $\begin{array}{c}\text { Desvio } \\
\text { Padrão } \\
\text { TBS }\left({ }^{\circ} \mathbf{C}\right)\end{array}$ \\
\hline $\mathbf{6 0}$ a 70 anos & 27,4 & 27,5 & 1,5 \\
$\mathbf{7 1}$ a 80 anos & 27,5 & 27,6 & 1,4 \\
$\mathbf{8 1}$ a 90 anos & 27,4 & 27,4 & 1,4 \\
Maior de 90 anos & 25,5 & 25,5 & 1,0 \\
\hline
\end{tabular}

As estatísticas das variáveis ambientais temperatura de bulbo seco (TBS), temperatura de globo $\left(\mathrm{T}_{\mathrm{g}}\right)$, temperatura radiante média (TRM), umidade relativa do ar (UR) e velocidade do ar $\left(\mathrm{V}_{\mathrm{ar}}\right)$ estão apresentadas na Tabela 14

Os resultados da análise da associação entre as variáveis ambientais e a variável "grau de sensação térmica", obtidos na ANOVA, tiveram a hipótese $\left(\mathrm{H}_{0}\right)$ de igualdade de medianas (teste de Tukey-HSD) rejeitada para todas as combinações de associações. Assim, inferiu-se que as mudanças de sensação térmica têm efeito com as mudanças de todas as variáveis ambientais.

Os resultados das associações das estimativas de probabilidade (probits) das respostas de sensação térmica (quente, confortável e frio), segundo as variáveis ambientais e a variável vestimenta, determinaram os parâmetros de conforto térmico para pessoas idosas na cidade de Campina Grande. A variável individual "vestimenta" apresentou forte correlação com a variável "grau de sensação térmica", sendo considerada, por essa razão, na determinação dos parâmetros.

O gráfico da Figura 4, com os probits das respostas de sensação térmica segundo a temperatura de bulbo seco (TBS), mostra o procedimento para a determinação desses parâmetros.

Analisando a Figura 4, pode-se concluir que as curvas referentes a "frio" e "quente" representam o intervalo de transição entre confortável e não confortável. Por exemplo, a linha vertical que passa a $27,5^{\circ} \mathrm{C}$ indica $100 \%$ dos votos de sensação térmica dos participantes. Acima da interseção entre a curva que representa as respostas à sensação de frio e a linha tracejada têm-se 12,5\% de pessoas desconfortáveis devido ao frio. $\mathrm{O}$ intervalo entre as interseções das curvas de frio e quente representam 55\% de pessoas confortáveis e, abaixo da interseção da linha tracejada com a curva de quente, têm-se 32,5\% de pessoas desconfortáveis devido ao calor. Traçando uma linha imaginária partindo do ponto de interseção entre a linha horizontal de $50 \%$ e as curvas de probits (frio e quente), definiu-se a faixa de conforto térmico para a TBS, entre $25,4^{\circ} \mathrm{C}$ e $28,4^{\circ} \mathrm{C}$.

$\mathrm{O}$ gráfico das curvas de probits acumulados de respostas para quente, confortável e frio, segundo a vestimenta está apresentado na Figura 5. Analisando o comportamento da curva para a categoria frio, podese compreender que, para valores inferiores a 0,2 clo, as probabilidades de ocorrência da sensação frio são maiores. Semelhante interpretação pode ser feita quanto à curva para a categoria quente. Quanto maior for 0 valor do clo, maiores são as chances de ocorrência da sensação quente. Em outras palavras, a pessoa idosa sentirá mais frio quando estiver com roupas mais leves e mais calor com roupas mais pesadas. Esses resultados já eram previsíveis, porém a contribuição da pesquisa é em relação à definição de seus limites mínimos e máximos.

$\mathrm{O}$ mesmo procedimento foi realizado com a TRM, a UR e a $\mathrm{V}_{\mathrm{ar}}$, que resultou na determinação dos parâmetros apresentados na Tabela 15.

Os parâmetros de conforto térmico apresentados na Tabela 15 podem ter a seguinte interpretação: aqueles indivíduos que se encontram dentro dos limites determinados estão na situação de conforto térmico. Já aqueles em condições inferiores ou superiores aos limites estão na situação de desconforto térmico.

\section{Conclusões}

A presente pesquisa investigou a sensação térmica de pessoas idosas residentes na cidade de Campina Grande, PB, Brasil, com o objetivo de determinar parâmetros de conforto térmico para auxiliar no planejamento de ambientes residenciais.

Os parâmetros determinados correspondem aos limites térmicos aceitáveis para pessoas com idade superior a 60 anos e traduzem as preferências térmicas desse grupo etário. 
Partindo da afirmação de Panet (2018) sobre a ausência de outros parâmetros que representem as preferências térmicas desse grupo etário no universo da pesquisa, esses constituem os únicos dados, até o momento, capazes de determinar as condições ambientais para o conforto térmico no interior de edificações residenciais ventiladas naturalmente.

Tabela 14 - Resumo das estatísticas das variáveis ambientais

\begin{tabular}{l|c|c|c|c|c}
\hline & TBS $\left({ }^{\circ} \mathrm{C}\right)$ & TG $\left({ }^{\circ} \mathrm{C}\right)$ & TRM $\left({ }^{\circ} \mathrm{C}\right)$ & UR $(\%)$ & Var $(\mathrm{m} / \mathbf{s})$ \\
\hline Média & 27,39 & 27,36 & 27,35 & $\mathbf{5 9 , 2 1}$ & $\mathbf{0 , 2 8}$ \\
Mediana & 27,40 & 27,30 & 27,22 & $\mathbf{5 8 , 3 5}$ & 0,16 \\
Máxima & $\mathbf{3 0 , 8 0}$ & $\mathbf{3 1 , 3 0}$ & $\mathbf{3 2 , 6 0}$ & $\mathbf{8 3 , 0 0}$ & $\mathbf{0 , 8 5}$ \\
Mínima & $\mathbf{2 3 , 7 0}$ & 23,40 & $\mathbf{2 2 , 6 1}$ & $\mathbf{4 1 , 6 0}$ & $\mathbf{0 , 1 0}$ \\
D.P. & $\mathbf{1 , 4 7}$ & $\mathbf{1 , 5 6}$ & $\mathbf{1 , 8 7}$ & $\mathbf{8 , 6 7}$ & $\mathbf{0 , 2 3}$ \\
C.V. $(\%)$ & $\mathbf{5 , 3 8}$ & $\mathbf{5 , 7 0}$ & $\mathbf{6 , 8 6}$ & $\mathbf{1 4 , 6 4}$ & $\mathbf{8 1 , 5 1}$ \\
\hline
\end{tabular}

Figura 4 - Gráfico das curvas de probits acumulados de respostas para quente, confortável e frio segundo a temperatura de bulbo seco (TBS)

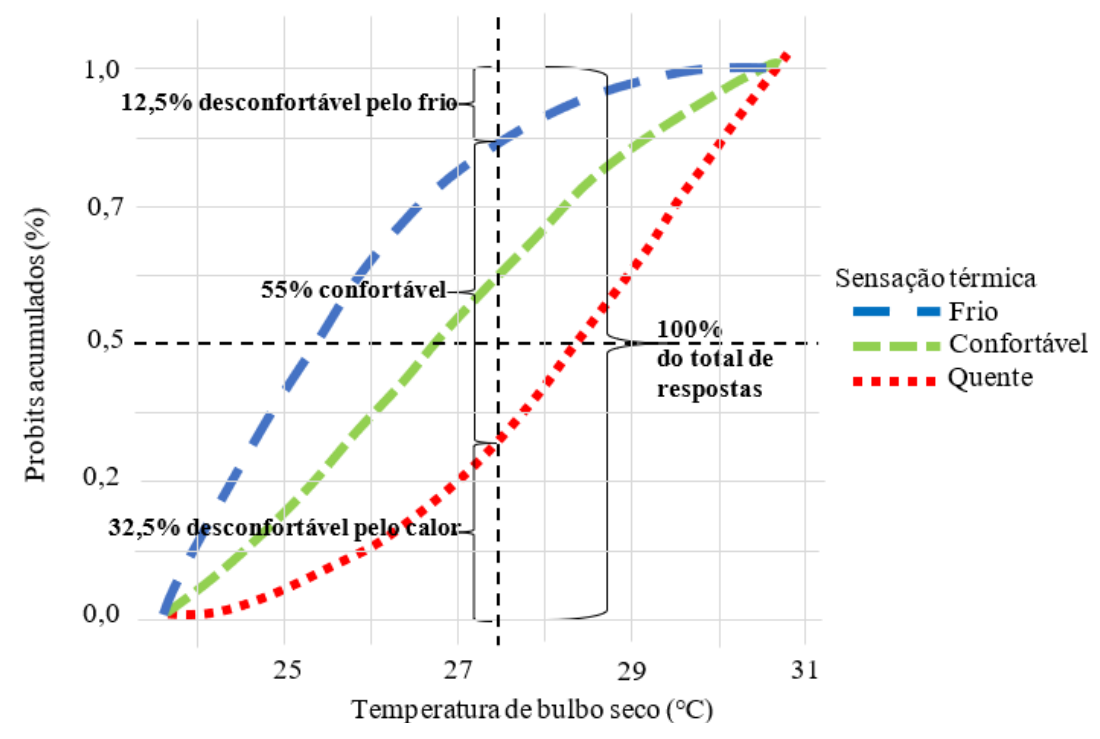

Figura 5 - Gráfico das curvas de probits acumulados de respostas para quente, confortável e frio segundo a vestimenta (clo)

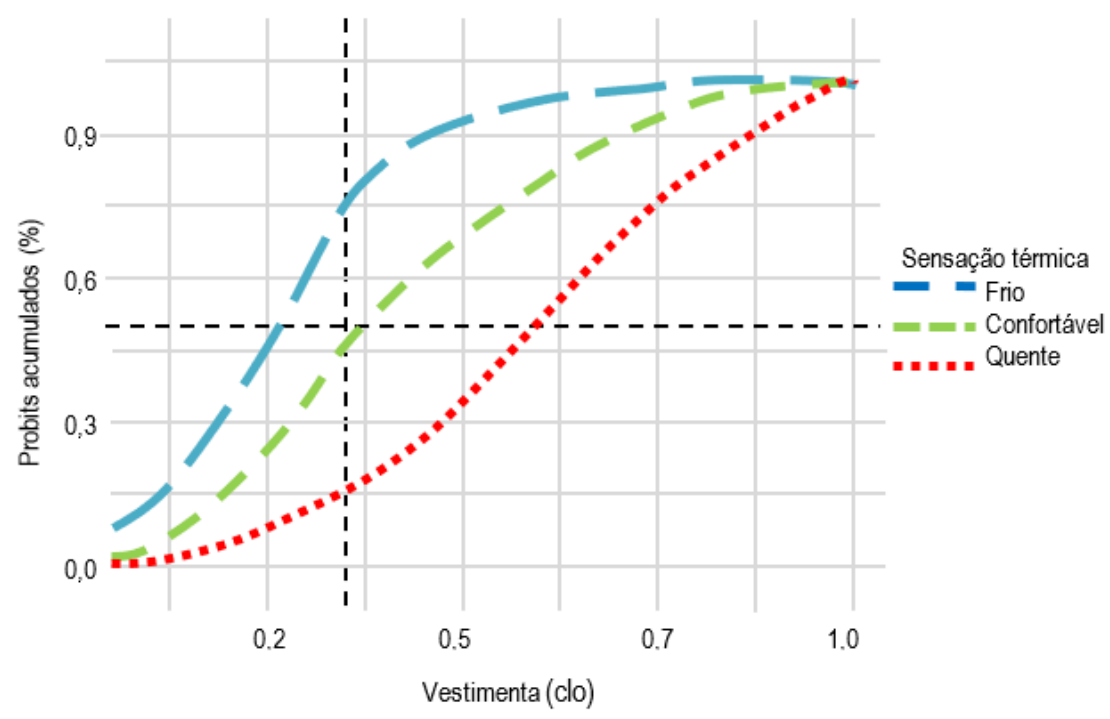

146 Panet, M. de F.; Araújo, V. M. D. de; Araújo, E. H. S. de 
Tabela 15 - Parâmetros de conforto térmico

\begin{tabular}{l|c}
\hline \multicolumn{1}{c|}{ Variáveis estudadas } & Parâmetros \\
\hline Temperatura de bulbo seco (TBS) & $25,4{ }^{\circ} \mathrm{C}-28,4{ }^{\circ} \mathrm{C}$ \\
Temperatura radiante média (TRM) & $26,4^{\circ} \mathrm{C}-27,8{ }^{\circ} \mathrm{C}$ \\
Umidade relativa do ar (UR) & $57,5 \%-62 \%$ \\
Velocidade do ar $\left(\mathrm{V}_{\mathrm{ar}}\right)$ & $0,3 \mathrm{~m} / \mathrm{s}-0,38 \mathrm{~m} / \mathrm{s}$ \\
Vestimenta & $0,25 \mathrm{clo}-0,60 \mathrm{clo}$ \\
\hline
\end{tabular}

Confirmando a hipótese apresentada na introdução, os resultados dos parâmetros determinados mostraram que a preferência térmica das pessoas idosas tende a ambientes mais aquecidos. Essa conclusão vai ao encontro de outros trabalhos que apresentam tendência ao declínio da sensibilidade térmica maior para as condições de calor (GUERGOVA; DUFOUR, 2011; DUFOUR; CANDAS, 2007). Neste caso, pode-se dizer que a preferência por ambientes mais aquecidos será influenciada pela redução da sensibilidade do corpo ao calor. No entanto, para as pessoas idosas esse fato pode ser preocupante se se considerar o comprometimento do desempenho do sistema passivo e dos órgãos do corpo humano (NOVIETO, 2013), principalmente em condições térmicas extremas.

Além disso, a presente pesquisa revelou a necessidade de estudos semelhantes, com o mesmo grupo etário, em regiões climáticas distintas, além da proposição de modelos de conforto térmico que considerem as particularidades das pessoas idosas.

Em estudos futuros pretende-se aperfeiçoar os resultados com tempo de duração do estudo observacional maior que 12 meses e coleta de dados nos vários turnos.

\section{Referências}

ARAÚJO, V. M. D. de. Parâmetros de conforto térmico para usuários de edificações escolares no litoral nordestino brasileiro. São Paulo, 1996. Tese (Doutorado em Arquitetura e Urbanismo) - Faculdade de Arquitetura e Urbanismo, Universidade de São Paulo, São Paulo, 1996.

BRASIL. Ministério da Saúde. Secretaria de Atenção à Saúde. Departamento de Atenção Básica. Manual de estrutura física das unidades básicas de saúde: saúde da família. Brasília: Ministério da Saúde, 2006. (Série A. Normas e Manuais Técnicos).

CHINDAPOL, S. et al. Thermal stress and comfort in elderly people's housing in tropical climates: the need for policy. Barcelona: World SB 14, 2014.

DUFOUR, A.; CANDAS, V. Ageing and thermal responses during passive heat exposure: sweating and sensory aspects. European Journal of Applied Physiology, v. 100, p. 19-26, 2007.

FERREIRA, M. S. Um modelo do sistema térmico do corpo humano.São Paulo, 2001. Tese (Doutorado em Engenharia Mecânica) - Escola Politécnica, Universidade de São Paulo, São Paulo, 2001.

FIALA, D. Dynamic simulation of human heat transfer and thermal comfort. Leicester, 1998. Thesis (Doctorate) - Institute of Energy and Sustainable Development, Montfort University, Leicester, 1998.

GUERGOVA, S.; DUFOUR, A. Thermal sensitivity in the elderly: a review. Ageing Research Reviews, v. 10, p. 80-92, 2011.

GÜNES, U. Y.; ZAYBAK, A. Does the body temperature change in older people? Journal of Clinical Nursing, v. 17, p. 2284-2287, 2008.

HANSEN, A. et al. Perceptions of heat-susceptibility in older persons: barriers to adaptation. International Journal of Environmental Research and Public Health, v. 8, p. 4714-4728, 2011.

INSTITUTO BRASILEIRO DE GEOGRAFIA E ESTATÍSTICA. Projeção da população do Brasil por sexo e idade 1980-2050. Rio de Janeiro: Estudos e Pesquisas, 2008. (Informação demográfica e socioeconômica, 24).

INSTITUTO NACIONAL DE METEOROLOGIA. Climatologia de meses e trimestres de maiores e menores temperaturas e pluviosidades médias no período de 1961-2009. Disponível em: http://www.inmet.gov.br/portal/. Acesso em: 14 maio 2016. 
INSTITUTO NACIONAL DE METEOROLOGIA. Climatologia de meses e trimestres de maiores e menores temperaturas e pluviosidades médias no período de 1981-2010. Disponível em: http://www.inmet.gov.br/portal/. Acesso em: 07 jul. 2018a.

INSTITUTO NACIONAL DE METEOROLOGIA. Dados históricos. Disponível em: http://www.inmet.gov.br/portal/. Acessoem: 07 jul. 2018b.

INTERNATIONAL STANDARDIZATION ORGANIZATION.ISO 7726: ergonomics of the thermal environment: instruments for measuring physical quantities. Genève, 1998.

INTERNATIONAL STANDARDIZATION ORGANIZATION. ISO 7730: ergonomics of the thermal environment: analytic determination and interpretation of thermal comfort using calculation of the PMV and PDD indices and local thermal comfort criteria.Genève, 2005.

INTERNATIONAL STANDARDIZATION ORGANIZATION. ISO 8996: ergonomics: determination of metabolic rate. Genève, 2004.

INTERNATIONAL STANDARDIZATION ORGANIZATION. ISO 9920: ergonomics of the thermal environment: estimation of thermal insulation and water vapour resistance of a clothing ensemble. Genève, 2007.

KENNEY, W. L; MUNCE, T. A. Invited review: aging and human temperature regulation. Journal of Applied Physiology, v. 95, p. 2598-2603, 2003.

KINGMA, B. R. M.; SCHWEIKER, A. W.; LICHTENBELT, W. D Van M. Exploring internal body heat balance to understand thermal sensation. Building Research \& Information, v. 45, n. 7, p. 808-818, 2017.

LIPSCHITZ, D. A. Screening for nutritional status in the elderly. Primary Care, v. 21, p. 55-67, 1994.

NOVIETO, D.T. Adapting a human thermoregulation model for predicting the thermal response of older persons. Leicester, 2013. Thesis (Doctorate) - Montfort University, Leicester, 2013.

NOVIETO, D. T; ZHANG, Y. Year: thermal comfort implications of the aging effect on metabolism, cardiac output and body weight. In: NOVIETO, D. T; ZHANG, Y. (Ed.).Adapting to change: new thinking on comfort. London: Network for Comfort and Energy use in Buildings, 2010.

OLIVEIRA, A. C. de. A influência das recomendações do zoneamento bioclimático brasileiro no desempenho térmico da envoltória de edificações de interesse social nos municípios da Paraíba. João Pessoa, 2013. Dissertação (Mestrado em Arquitetura e Urbanismo) - Centro de Tecnologia, Universidade Federal da Paraíba, João Pessoa, 2013.

PANET, M. de F. Um futuro confortável: modelagem preditiva de sensação térmica de pessoas idosas em localidade do semiárido paraibano. Natal, 2018. 151f. Tese (Doutorado em Arquitetura e Urbanismo) Centro de Tecnologia, Universidade Federal do Rio Grande do Norte, Natal, 2018.

RORIZ, M. Uma proposta de revisão do Zoneamento Bioclimático Brasileiro. ANTAC - Associação Nacional de Tecnologia do Ambiente Construído. São Carlos, 2012. Disponível em: < http://www.labeee.ufsc.br/sites/default/files/projetos/Proposta_Revisao_Zoneamento_Bi oclimatico.pdf>. Acessoem: 5 mai. 2015.

TSUZUKI, K.; OHFUKU, T. Thermal sensation and thermoregulation in elderly compared to young people in Japanese winter season. Indoor Air, 2002.

VILLA, S. B.; ORNSTEIN, S. W (Org.). Qualidade ambiental na habitação: avaliação pós-ocupação.São Paulo: Oficina de Textos, 2013.

WILSON, T. et al. Effect of aging on cardiac function during cold stress in humans. TheAmerican JournalofPhysiology- Regulatory, Integrative and ComparativePhysiology, v. 298, p.1627-1633, 2010.

WORLD HEALTH ORGANIZATION. World report on ageing and health. Geneva: World Health Organization, 2015. 


\section{Miriam de Farias Panet}

Unidade Acadêmica de Engenharia Civil | Universidade Federal de Campina Grande | Rua Aprígio Veloso, 882, Universitário | Campina Grande - PB - Brasil | CEP 58428-830 | Tel.: (84) 99984-1960 | E-mail: miriampanet@gmail.com

Virgínia Maria Dantas de Araújo

Departamento de Arquitetura | Universidade Federal do Rio Grande do Norte | Av. Sen. Salgado Filho, 3000, Candelária | Natal - RN Brasil | CEP 59078-970 | Tel.: (84) 99401-0160 | E-mail: virginiamdaraujo@gmail.com

Eduardo Henrique Silveira de Araújo

Departamento de Demografia e Ciências Atuariais | Universidade Federal do Rio Grande do Norte | Tel.: (84) 99982-6065 | E-mail: ehsa@ccet.ufrn.br

\section{Ambiente Construído}

Revista da Associação Nacional de Tecnologia do Ambiente Construído

Av. Osvaldo Aranha, $99-3^{\circ}$ andar, Centro

Porto Alegre - RS - Brasil

CEP $90035-190$

Telefone: +55 (51) 3308-4084

Fax: +55 (51) 3308-4054

www.seer.ufrgs.br/ambienteconstruido

E-mail: ambienteconstruido@ufrgs.br 